\title{
Design and Evaluation of Modifications to the NASA Langley Flow Impedance Tube
}

\author{
M. G. Jones, ${ }^{*}$ W. R. Watson,${ }^{\dagger}$ T. L. Parrott ${ }^{\ddagger}$ \\ NASA Langley Research Center, Hampton, VA 23681-2199, USA \\ and \\ C. D. Smith ${ }^{\S}$ \\ Lockheed Martin Engineering and Sciences, Hampton, VA 23681-2199, USA
}

\begin{abstract}
The need to minimize fan noise radiation from commercial aircraft engine nacelles continues to provide an impetus for developing new acoustic liner concepts. If the full value of such concepts is to be attained, an understanding of grazing flow effects is crucial. Because of this need for improved understanding of grazing flow effects, the NASA Langley Research Center Liner Physics Group has invested a large effort over the past decade into the development of a 2-D finite element method that characterizes wave propagation through a lined duct. The original test section in the Langley Grazing Incidence Tube was used to acquire data needed for implementation of this finite element method. This test section employed a stepper motor-driven axial-traversing bar, embedded in the wall opposite the test liner, to position a flush-mounted microphone at pre-selected locations. Complex acoustic pressure data acquired with this traversing microphone were used to educe the acoustic impedance of test liners using this 2-D finite element method and a local optimization technique. Results acquired in this facility have been extensively reported, and were compared with corresponding results from various U.S. aeroacoustics laboratories in the late 1990's. Impedance data comparisons acquired from this multi-laboratory study suggested that it would be valuable to incorporate more realistic 3-D aeroacoustic effects into the impedance eduction methodology. This paper provides a description of modifications that have been implemented to facilitate studies of 3-D effects. The two key features of the modified test section are (1) the replacement of the traversing bar and its flush-mounted microphone with an array of 95 fixed-location microphones that are flush-mounted in all four walls of the duct, and (2) the inclusion of a suction device to modify the boundary layer upstream of the lined portion of the duct. The initial results achieved with the modified test section are provided in this report, and a comparison of these results with those achieved using the original test section is used to demonstrate that the data acquisition and analysis with the new test section can be confidently used for impedance eduction.
\end{abstract}

\section{Nomenclature}

$c_{0} \quad=$ ambient sound speed, $\mathrm{m} / \mathrm{s}$

$H, L \quad=$ test section height and length, respectively, $\mathrm{m}$

$i=\sqrt{-1}$

$k \quad=$ free space wavenumber, $\mathrm{m}^{-1}$

$L_{1}, L_{2} \quad=$ axial locations of leading and trailing edges of liner, respectively, $\mathrm{m}$

$M \quad=$ Mach number

$p \quad=$ complex acoustic pressure, $\mathrm{Pa}$

$p_{s} \quad=$ acoustic pressure at source plane, $\mathrm{Pa}$

$P O A=$ percent open area, $\%$

$x, y, z \quad=$ axial, vertical and spanwise locations, respectively, $\mathrm{m}$

$x_{i} \quad=$ wall measurement axial location, $\mathrm{m}$

\footnotetext{
* Research Scientist, Structural Acoustics Branch, Mail Stop 463; Michael.G.Jones@NASA.GOV. Senior member of AIAA.

${ }^{\dagger}$ Senior Research Scientist, Computational Modeling and Simulation Branch, Mail Stop 128; Willie.R.Watson@NASA.GOV. Senior member of AIAA.

¥Senior Research Scientist, Structural Acoustics Branch, Mail Stop 463; Tony.L.Parrott@NASA.GOV.

${ }^{\S}$ Staff Aeronautical Engineer, Mail Stop 105; C.S.Smith@LaRC.NASA.GOV.
} 


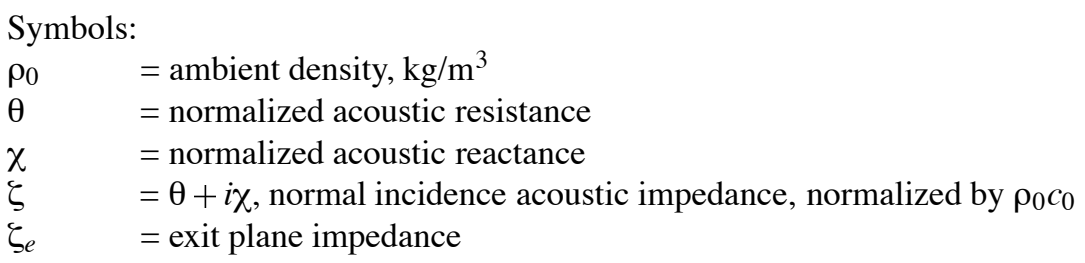

\section{Introduction}

$\mathrm{T}_{\mathrm{i}, \mathrm{m}}$ HE continuing need to minimize fan noise radiation from commercial aircraft engine nacelles has provided an impetus for testing a number of new acoustic liner concepts. Although locally-reacting acoustic liners with uniform impedance are still commonly used to achieve significant noise reductions, the trend toward shorter and wider inlets is compelling liner designers to seek more efficient, advanced liner technologies. Whether these are uniform or nonuniform impedance liners, ${ }^{1,2}$ it is increasingly crucial that the effects of grazing flow be accurately quantified. To that end, a number of impedance prediction models have been developed over the last five decades to predict the impedance of locally-reacting acoustic liners, both with and without grazing flow. ${ }^{3-6}$

The NASA Langley Research Center Liner Physics Group has emphasized grazing flow impedance eduction technology over the past three decades. ${ }^{7,8}$ The original Langley Grazing Incidence Tube (GIT) incorporated a stepper motor-driven axial-traversing bar, embedded in the wall opposite the test liner, to position a flush-mounted microphone at pre-selected locations. From the complex acoustic pressure data acquired with this microphone, the liner impedance is educed using (1) an analytical approach based on the assumption of a single, dominant, propagating mode, ${ }^{6}$ or (2) a 2-D finite element method (FEM) that characterizes wave propagation through the lined duct. ${ }^{9}$ Based in part on the results of a comparison study of impedance eduction techniques used by various U.S. aeroacoustics laboratories, ${ }^{8}$ NASA Langley decided to further develop the FEM to incorporate realistic 3-D aeroacoustic effects, and to implement significant modifications to the GIT. The purpose of this paper is to describe these modifications, and to provide a status (pro and con) regarding the enhancements resulting from their implementation. The enhancements consist of (1) increased testing efficiency, (2) increased frequency range and (3) inclusion of boundary layer control. Finally, a comparison of impedances educed for two liners using the original and modified GIT test sections is provided to demonstrate consistency of results.

\section{GIT Test Sections}

number of enhancements have been incorporated into the new GIT test section. Sketches of the original and Amodified GIT test sections are provided in figures 1 and 2. These two test sections are installed in the same flow duct, with the original test section located approximately $2 \mathrm{~m}$ downstream of the modified test section. For convenience, the original and modified test sections will be designated GIT-TB and GIT-95M, respectively, to reflect their key difference (TB for traversing bar, 95M for 95 fixed-location microphones). Each test section has internal dimensions of $0.812 \mathrm{~m} \times 0.051 \mathrm{~m} \times 0.51 \mathrm{~m}$, and contains an axially-centered test liner with a maximum length of 0.406 $\mathrm{m}$ (test liner length is 8 duct diameters, with hardwall section lengths of 4 duct diameters upstream and downstream of liner). The surface of the test liner forms the bottom wall for the GIT-TB or the upper wall for the GIT-95M. Elsewhere, the test section side-walls are rigid, with a source upstream of the liner and a near-anechoic termination downstream of the liner.

There were a number of hardware and software (analysis) issues with the GIT-TB. Some of the key issues were:

1. Data acquisition with a single traversing microphone for a complete liner test series (e.g., four centerline Mach numbers and six source frequencies, for a total of 24 test conditions) typically requires approximately 2.5 days of testing. This necessitates multiple iterations of test facility cycling (startup and shutdown). Clearly, as the number of facility operational cycles increases, the achievement of identical operating conditions for the complete test series becomes even more difficult.

2. The usage of a traversing bar introduces leakage along the test section wall containing the traversing bar, and may be the cause of the observed mean flow profile distortion that is present over the lined surface. 


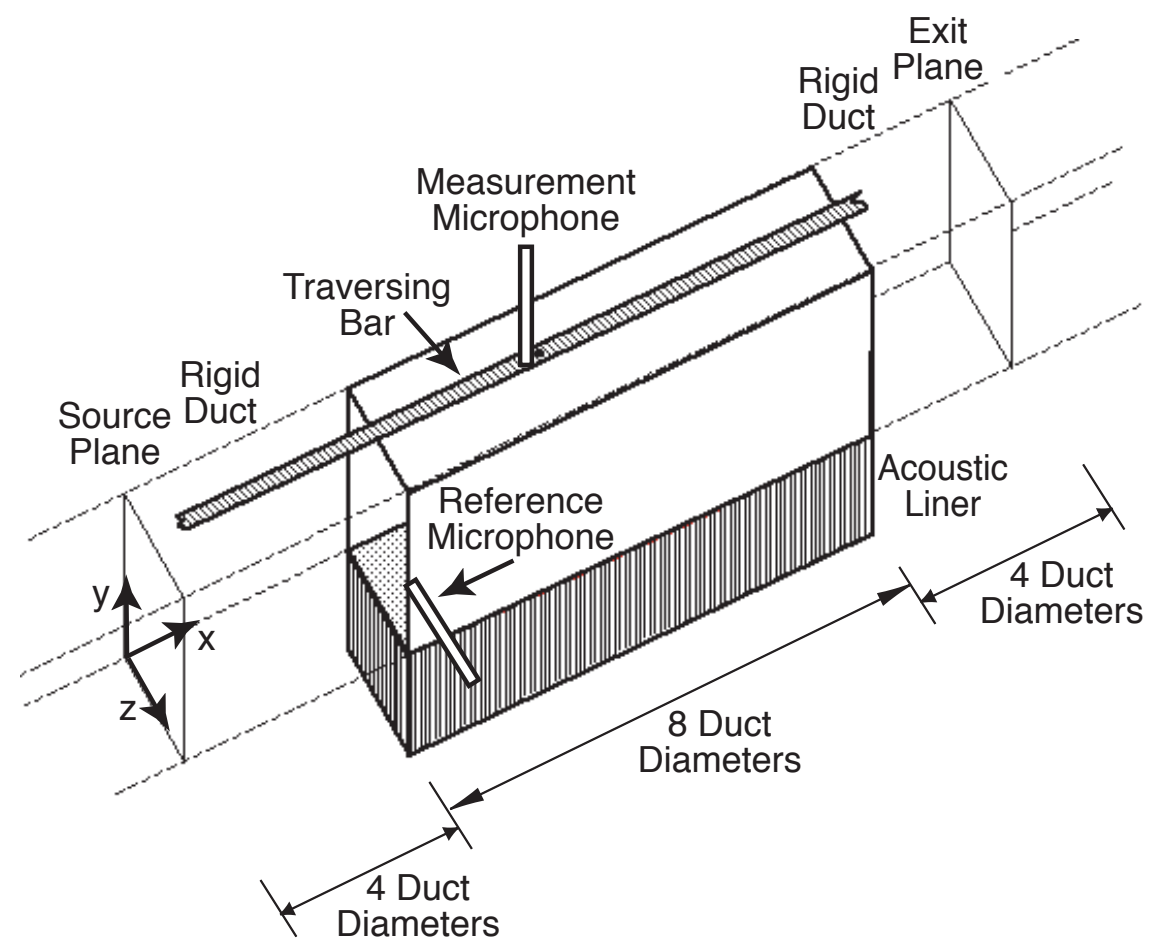

Figure 1. Original test section (GIT-TB), with test liner centered in axial span of test section.

3. Impedance eduction methods are limited to excitation frequencies below cut-on in the hardwall section at the source plane (approximately $3 \mathrm{kHz}$ ). This limitation results from the use of a 2-D FEM and the restriction of acoustic pressure measurements to a single axial array of locations. Extension to higher frequencies will require inclusion of higher-order mode effects in the FEM (i.e., incorporation of 3-D FEM).

4. Flow profiles are characterized by near fully-developed turbulent duct flow with no independent boundary layer control. Thus, the effects of boundary layer shape and thickness cannot be studied. Also, previous methods ${ }^{9}$ typically relied upon 2-D analysis, in which the mean flow profile is assumed to vary in only the axial and vertical (from hardwall to liner) directions.

The above issues with the GIT-TB are addressed in the GIT-95M as follows:

1. The GIT-95M is placed upstream of the GIT-TB by $2 \mathrm{~m}$ (see figure 3 ), and the GIT-TB traversing bar is replaced with 95 flush-mounted microphones that are distributed on all four test section walls. Their locations were selected to achieve the following goals:

(a) An overriding requirement was to maintain an ability to use the 2-D FEM and single mode method (SMM) impedance eduction methodologies (same as used with the GIT-TB) with an appropriate subset of the GIT-95M microphone locations. By maintaining this capability, the data acquired with the GIT-95M can be properly compared with corresponding data acquired with the GIT-TB. This will allow the extensive database from previous tests to be used together with data acquired in the new test window to more fully understand the underlying physics of grazing flow effects on acoustic impedance. As will be shown in the "Results and Discussion" section, data acquired with the 31 microphones mounted on the lower wall (opposite the liner) were used as input to the 2-D FEM to compare with results achieved in the GIT-TB (using the traversing bar system). These comparisons will demonstrate that this capability to continue to use the 2-D FEM with the fixed-location microphones in the GIT-95M has been achieved. Since previous comparisons between the 2-D FEM and the SMM impedance eduction methodologies ${ }^{9}$ showed the two methods to be well matched, the results with the 2-D FEM also imply that the SMM can be successfully used with the GIT-95M.

(b) Four flush-mounted microphones were positioned in two axial planes (total of eight microphones), separated by $25.4 \mathrm{~mm}$, at the source end of the test section (see figure 2). Acoustic pressure measurements 


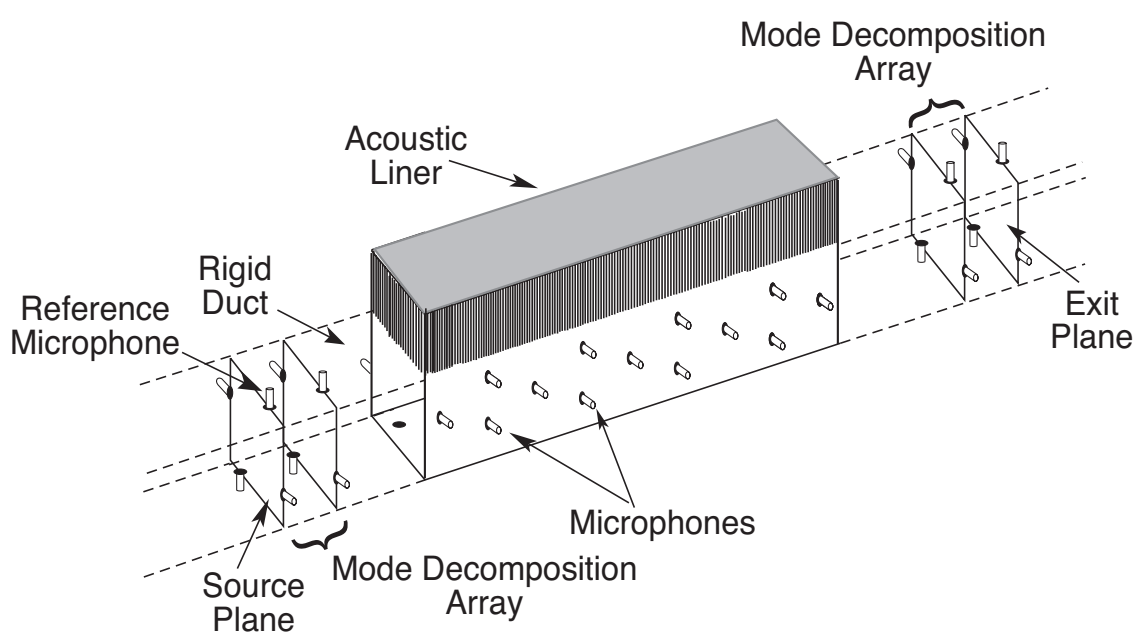

Figure 2. Modified test section (GIT-95M).

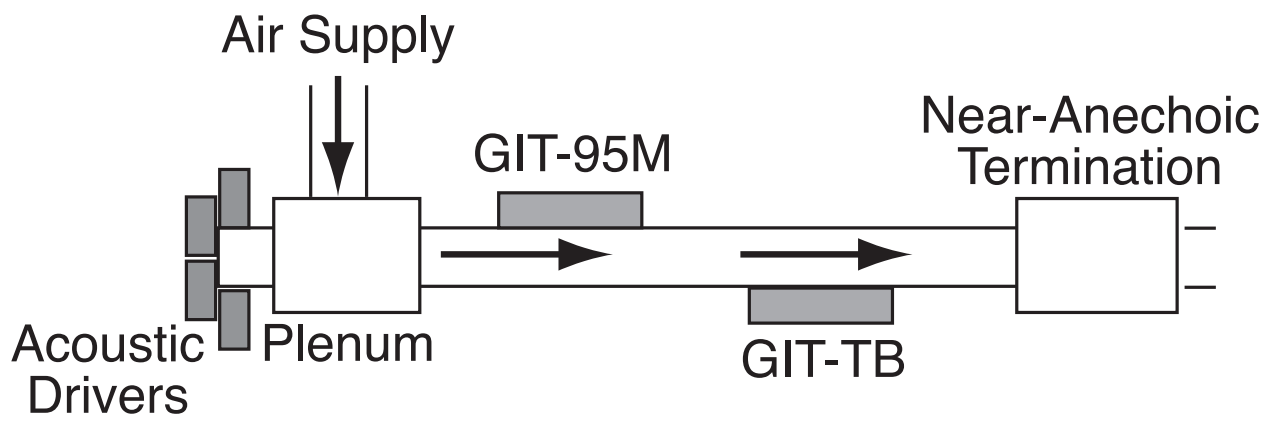

Figure 3. Sketch of grazing incidence tube, with both test windows.

were obtained at four points around the periphery of each plane for the purpose of performing modal decomposition at frequencies up to $10 \mathrm{kHz}$. A similar microphone location pattern was used at the exit end of the test section, such that the modal content at the exit plane can also be educed. For the current paper, however, the frequencies are sufficiently low (up to $3 \mathrm{kHz}$ ) that no higher order modes are cut on in the hardwall sections. Thus, this mode decomposition methodology will be discussed in future reports.

(c) The remainder of the microphones were flush-mounted in the four walls of the test section. Their locations were chosen such that the complex acoustic pressure distributions at the duct walls could be adequately resolved up to $10 \mathrm{kHz}$.

A new data acquisition system, capable of simultaneously acquiring data with 48 microphones, was implemented for the GIT-95M. Agilent analog-to-digital devices are used to simultaneously acquire the data for 48 channels, and a Cytec switch matrix is used to switch from one set of 48 microphones (reference microphone plus microphones 2 through 48 ) to another set of 48 microphones (reference microphone plus microphones 49 through 95). The reference microphone (one of the 95 flush-mounted microphones, located in the source plane) provides the common basis for both sets of measurements, such that measurements at all 95 locations can be properly compared.

For each data acquisition, 2000 averages on each microphone channel (blocks of 2048 data points per average) were used in the current investigation. To reduce the influence of flow noise, a cross-spectrum signal extraction method ${ }^{10}$ was then used to determine the amplitudes and phases at each of the microphone locations (relative to the amplitude and phase at the reference microphone location). Clearly, fewer averages are needed for tests in which the signal-to-noise ratio is high. However, as the mean flow velocity is increased, the flow noise reduces 
the signal-to-noise ratio. Also, if the liner being investigated provides sufficient absorption of the test signal (a single tone source was used in the current investigation), the signal-to-noise ratio correspondingly decreases at the downstream microphone locations. Of course, the absorption of the test liner is not known a priori, so the number of averages must be predetermined without this knowledge. Based on previous experience with the liners included in the current study, it was determined that 2000 averages would be more than sufficient to ensure quality data.

A complete data acquisition of all 95 microphones thus consists of two sets of 2000 averages (one for each set of 48 microphones). This data is acquired and stored on the computer for post-processing in approximately 10 minutes. This reduces the time required for a complete liner test series by a factor of 5 (from 2.5 to 0.5 days). It should be noted that the data acquisition process, in which the traversing bar microphone was sequentially positioned at each test location for measurement, was the major time component for liner tests in the GIT-TB. As a result, the associated time required to set up for each desired mean flow velocity was of minimal concern. Conversely, the increased efficiency of the new data acquisition process is such that the time required for a liner test in the GIT-95M is dominated by this mean-flow set up time. In fact, it is possible to significantly increase the number of test frequencies with minimal impact on the overall test time. This will allow more detailed study of liner concepts in the future. However, for the purposes of this paper, the same frequencies as were previously used will be presented in order to allow direct comparison between the results acquired with each test section.

2. There is no leakage in the GIT-95M test section, because it has no moving parts. This enhancement has multiple desirable consequences. Obviously, if the mean flow profile is not distorted due to leakage, the parallel-flow assumption can be used with increased confidence. Also, the elimination of leakage in the system will allow future tests to be conducted at static pressures that are significantly different from the atmospheric pressure in the test facility. Historically, all tests have been conducted with the static pressure in the test section (GIT-TB) nearly the same as the atmospheric pressure, such that leakage into or out of the test section was minimized. The elimination of this requirement is expected to allow testing at higher Mach numbers than have been previously achievable.

3. The NASA Langley FEM impedance eduction method is being upgraded to incorporate 3-D aeroacoustic effects. A no-flow version has been recently evaluated using the modified test section data. A genetic algorithm is used to improve the accuracy (i.e., the ability to find the global optimum) of the optimization, and parallelization is used to achieve efficient implementation of this 3-D FEM. As expected, the impedances educed with the 3-D FEM are well-matched to those educed using the 2-D FEM (all for no-flow condition). However, this discussion is outside the scope of the current report; thus, detailed results of 3-D FEM analysis will be presented in future reports. In the current paper, the data were processed with the 2-D finite element method, and comparisons between results achieved with the same liners in the two test sections (GIT-TB and GIT-95M) will be presented.

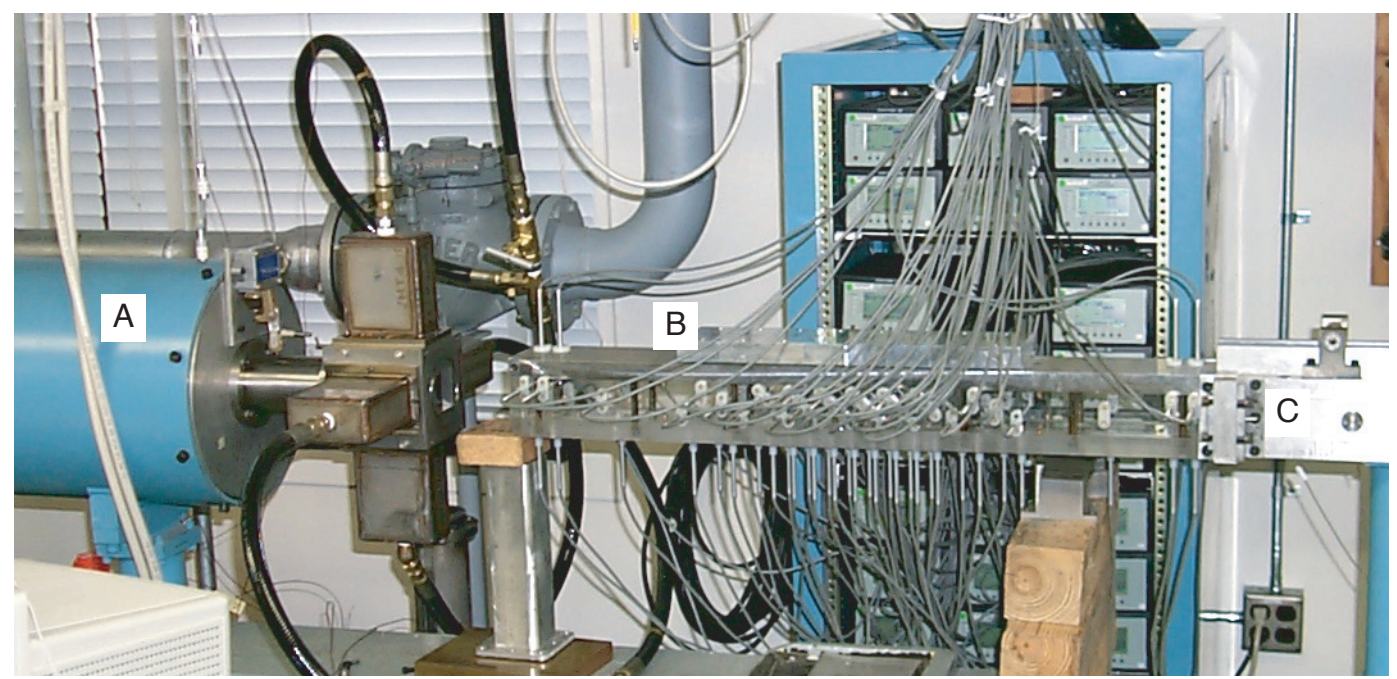
A. Air source plenum
B. Top-mounted liner fixture
C. Termination

Figure 4. Photograph of GIT-95M. 
4. Boundary layer control is being implemented just upstream of the GIT-95M (see figure 4) to allow suction over an axial length of $10 \mathrm{~mm}$ on two opposite walls (two sides or top and bottom) or on all four walls. This is expected to allow the study of altered mean flow profiles. The effects of boundary layer growth over the length of the liner can also be investigated. Preliminary tests were conducted for inclusion in the current report, but the results showed that the limited amount of suction applied to the boundary layer was insufficient. As a result, the flow profile did not change enough to warrant further acoustic studies at the present time. This issue will be pursued in future studies. The GIT-95M has also been designed to allow the suction devices to be replaced with blowing devices, such that the boundary layer can be thickened in a controlled manner. It is hoped that a comparison of these three types of tests (standard, with suction, with blowing) can be used to better understand the effects of boundary layer thickness on the acoustic performance of conventional liners.

Table 1. Conventional acoustic liner configurations.

\begin{tabular}{|c||c|c|}
\hline Configuration & 1 & 2 \\
\hline POA $(\%)$ & 6.4 & 15.0 \\
\hline Hole Diameter $(\mathrm{mm})$ & 0.99 & 0.99 \\
\hline Sheet Thickness $(\mathrm{mm})$ & 0.64 & 0.64 \\
\hline Cavity Depth $(\mathrm{mm})$ & 38.1 & 38.1 \\
\hline
\end{tabular}

\section{Description of Test Liners}

$\mathrm{T}$

WO types of acoustic liners were used in the current study; ceramic tubular and conventional perforate. The ceramic tubular material was chosen because its resistance is dominated by internal fluid viscosity and is thus very insensitive to mean flow effects. Conversely, sensitivity to mean flow velocity is significant for the conventional perforate liner due to vortex generation in the vicinity of the orifices. The two liner types, shown schematically in figure 5, are described in detail here:

(a)
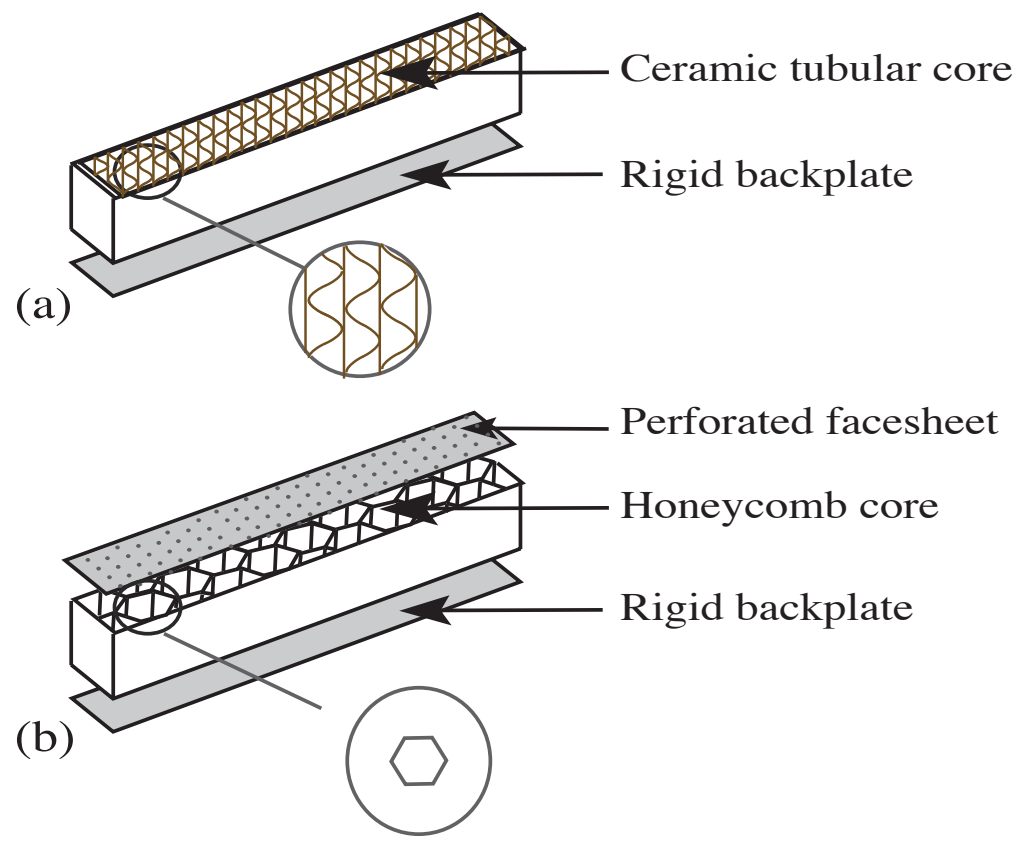

Figure 5. Sketch of test liners; (a) ceramic tubular, (b) conventional perforate.

a) The ceramic tubular liner consists of "sinusoid-shaped" parallel channels embedded in a ceramic matrix. These channels, with equivalent circular diameters of $0.76 \mathrm{~mm}$, run perpendicular to the exposed surface and provide 
a surface porosity of $65 \%$. The $77.5 \mathrm{~mm}$-deep channels are rigidly terminated such that each is isolated from its neighbor to ensure a locally reacting structure. The channel diameter is small enough that the grazing flow effects are insignificant relative to internal viscous losses.

b) Each perforate liner consists of an aluminum facesheet bonded onto $9.5 \mathrm{~mm}$-diameter hexcell honeycomb cavities that are $38.1 \mathrm{~mm}$ in depth. Critical geometric parameters of these two liners are provided in table 1 . These liners are modeled as lumped element single-degree-of-freedom, resonant systems with the resistance component supplied mostly by fluid dynamical vortex shedding associated with acoustically driven fluid oscillation in the perforated holes with length-to-diameter ratios of 0.65 . In contrast with the ceramic tubular structure, this fluid mechanical resistance is greatly influenced by grazing flow. These liners are similar to those currently installed in commercial aircraft engines for noise suppression.

\section{Test Description}

$\mathrm{T}^{\mathrm{s}}$ methodology for acquiring data with the GIT-TB has been thoroughly described in previous reports; ${ }^{8,9}$ therefore, the following will document only the revised test description associated with the GIT-95M. This GIT-95M test sequence consists of four major elements: (1) amplitude calibration of the reference microphone, (2) exit impedance eduction, (3) calibration of the 95-microphone system, and (4) complex acoustic pressure measurements at selected test conditions with the test liner installed in the duct. These data are then used to educe the impedance of the test liner as described in the following "Analysis" section.

\section{A. Reference Microphone Calibration}

$T^{H E}$ simplest of the test sequence steps is the calibration of the reference microphone. This microphone, which is 1 flush-mounted in the upper wall of the GIT-95M test section at the source plane $(x=0, y=H)$, is removed from the test section and calibrated using a $1000 \mathrm{~Hz}, 114 \mathrm{~dB}$ Bruel \& Kjaer calibrator. It is then mounted back into the GIT-95M test section.

\section{B. Exit Impedance Eduction}

$\mathbf{T}^{\mathrm{T}}$ is imperative that the exit impedance is known for each subsequent test condition. The exit impedance at one selected condition ( $1000 \mathrm{~Hz}$ source frequency, Mach 0 mean flow) is required for use in the in situ calibration of the remaining 94 microphones. The exit impedance spectra are also used as key boundary conditions in the 2-D FEM to educe the test liner impedance spectra.

A rotating plug containing two flush-mounted microphones was mounted into the sidewall of the GIT, approximately $1.5 \mathrm{~m}$ downstream of the GIT-95M exit plane. With a hardwall configuration (i.e., hardwall 'liner' mounted in the test section), the two microphone method ${ }^{11}$ was used to determine the impedance at the test section exit plane $(x=L)$ for each possible test condition (each combination of frequency and Mach number). A single-tone source with a reference SPL (sound pressure level at reference microphone location) of $130 \mathrm{~dB}$ was used for all tests, and the harmonics were sufficiently low (at least $10 \mathrm{~dB}$ below the level of the source frequency) that they could be ignored. These same data were also acquired with other liners installed, and the resultant exit impedances were compared with those acquired for the hardwall configuration. As expected, the impedances were nearly the same for both configurations. Thus, it was decided that the exit impedance educed with the hardwall configuration could be used for successive tests, regardless of the liner configuration.

\section{Calibration of 95 Microphone System}

$\mathrm{T}^{\mathrm{T}}$ is absolutely essential that an in situ process be used to calibrate the array of 95 microphones; else, the time 1 saved by the new data acquisition system would be completely lost. Clearly, if each microphone had to be removed and calibrated individually, this process would be very lengthy. Since calibration of both the amplitude and phase components is required for the analysis, individual microphone calibration would require the usage of a calibrator capable of providing both components. Such devices are available, but the individual calibration of 95 microphones using these devices would be a lengthy process. In addition, the handling of microphones (cycle of removal from duct, insertion in calibrator, removal from calibrator, insertion in duct) introduces potential for error into the calibration process. 
For these reasons, an in situ process was devised for calibration of the remaining 94 microphones (the reference microphone must still be calibrated individually). As discussed earlier, the exit impedance was educed for a hardwall configuration at a test condition of $1000 \mathrm{~Hz}$, with no mean flow. For this frequency, only planes waves are present throughout the test section. Thus, the amplitude and phase profiles down the length of the test section can be easily computed based on this exit impedance.

While the two-microphone exit impedance data was acquired for the calibration condition (Mach $0,1000 \mathrm{~Hz}$ ), the complex acoustic pressures were simultaneously acquired using each of the 95 microphones in the test section. These acoustic pressures were compared with the corresponding acoustic pressures that were predicted for each of the microphone locations based on the known source $(130 \mathrm{~dB}$, as measured with the reference microphone) and exit impedance boundary conditions. The differences between predicted and measured complex acoustic pressures were recorded and used as the calibration factors for all successive tests (with and without grazing flow) during that day (calibration process repeated each day prior to testing).

Finally, these calibration factors were compared to the corresponding calibration factors provided by the manufacturer for the microphone and signal conditioning system. Any microphone that was determined to be outside the acceptable error range (differences between predicted and measured becomes too large relative to manufacturer specifications) is not used in the successive analysis.

\section{Acoustic Liner Test Sequence}

TESTS were conducted with a discrete tone source (one tone at a time), at frequencies of 1000 to $3000 \mathrm{~Hz}$, in steps

of $500 \mathrm{~Hz}$. Each of these frequencies was tested at centerline Mach numbers of 0.0, 0.3 and 0.4. A function generator was used to supply a sinusoidal signal at the desired test frequency to four 120-watt acoustic drivers (see figure 3). The acoustic waves emitted from the acoustic drivers propagate from left to right across the surface of the test panel, and into a termination section designed to minimize reflections over the frequency range of interest. At each test frequency, the sound pressure level at the source plane (as measured by the reference microphone) was set to $130 \mathrm{~dB}$. The data acquisition system described earlier was used to read all 95 flush-mounted microphones in the GIT-95M test section, and the data were recorded for post-processing.

\section{Analysis}

$T_{\mathrm{p}}^{\mathrm{H}}$ HE 2-D finite element impedance eduction method (FEM) used in this study has been discussed at length in previous papers; ${ }^{12}$ thus, only a brief summary of the key details is provided in this report. Usage of the FEM with the GIT-95M test section is essentially the same as in the GIT-TB, with the only differences being the interchange of the liner location and the measurement ports. The applicable geometry and coordinate system used to model the GIT-TB test section for the FEM are depicted in figure 6. It is assumed that the mean flow profile is uniform and only plane acoustic waves exist in the spanwise direction (perpendicular to the $x$-y plane; not shown in the sketch). The maximum frequency $(3000 \mathrm{~Hz})$ is below the cut-on frequency for higher-order modes in a hardwall region for all conditions included in this investigation. In the lined section, the two sidewalls are hard; thus, the assumption that no spanwise higher-order modes carry energy is reasonable. As a result, the 2-D FEM can be used with confidence.

The regions of the computational domain upstream $\left(0 \leq x \leq L_{1}\right)$ and downstream $\left(L_{2} \leq x \leq L\right)$ of the liner contain rigid walls. As described earlier, the complex acoustic pressures are measured at each of the measurement locations along the upper wall (at $x=0, x_{1}, x_{2}, \ldots x_{i}$ ) using the microphone flush-mounted in the traversing bar. The FEM finds the solution to the steady-state form of the 2-D convected wave equation (using an $e^{i \omega t}$ time convention),

$$
\left(1-M^{2}\right) \frac{\partial^{2} p}{\partial x^{2}}+\frac{\partial^{2} p}{\partial y^{2}}-2 i k M \frac{\partial p}{\partial x}+k^{2} p=0
$$

The source plane acoustic pressure boundary condition is

$$
p=p_{s}
$$

where $p_{s}$ is the acoustic pressure profile in the vertical direction at the source plane. Because only plane waves are assumed at the source plane, $p_{s}$ is set to the constant value measured with the traversing microphone placed in the source plane.

The exit plane boundary condition is

$$
\frac{\partial p}{\partial x}=\frac{-i k p}{M+\zeta_{e}}
$$




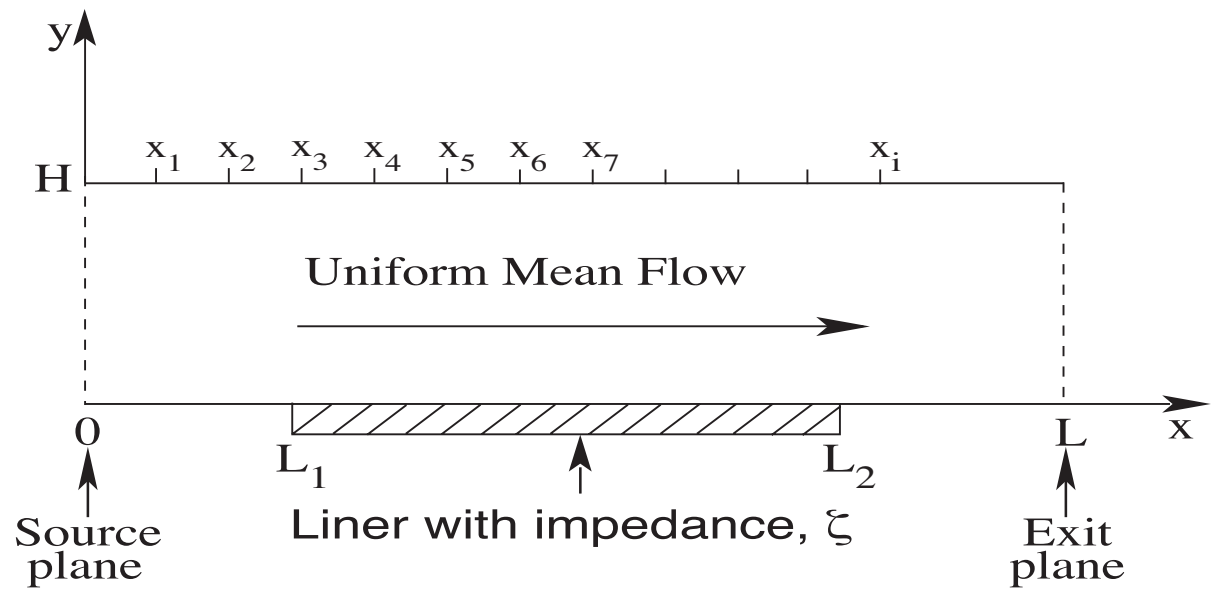

Figure 6. Computational domain for 2-D FEM (not to scale).

which is derived from the requirement that the exit impedance, $\zeta_{e}$, must equal the ratio of the acoustic pressure to the axial acoustic velocity in that plane. Since only plane waves are assumed to be present at the exit plane, $\zeta_{e}$ is taken to be the constant value determined using flush-mounted microphones and plane wave analysis ${ }^{11}$ (reference "Exit Impedance Eduction" section above).

The boundary conditions at all rigid walls are given as

$$
\frac{\partial p}{\partial y}=0
$$

which means the normal component of acoustic particle velocity vanishes at a rigid wall. Finally, the boundary condition for the lined region of the duct $\left(L_{1} \leq x \leq L_{2}\right.$ in figure 6$)$ is given by ${ }^{13}$

$$
\frac{\partial p}{\partial y}=\frac{i k p}{\zeta}+\frac{2 M}{\zeta} \frac{\partial p}{\partial x}+\frac{M^{2}}{i k \zeta} \frac{\partial^{2} p}{\partial x^{2}}
$$

Equations (1)-(5) constitute a boundary value problem that can be solved to obtain the upper wall complex acoustic pressure when the impedance of the liner, $\zeta$, is known. Alternatively, an iterative approach may be used to determine the liner impedance for which the measured and predicted upper wall complex acoustic pressures are matched to within a predetermined tolerance. In this approach, the difference between the measured and predicted (via 2-D FEM) is used as an objective function that is minimized to determine the unknown liner impedance. A Davidon-Fletcher-Powell optimization algorithm is used for this purpose. ${ }^{14}$
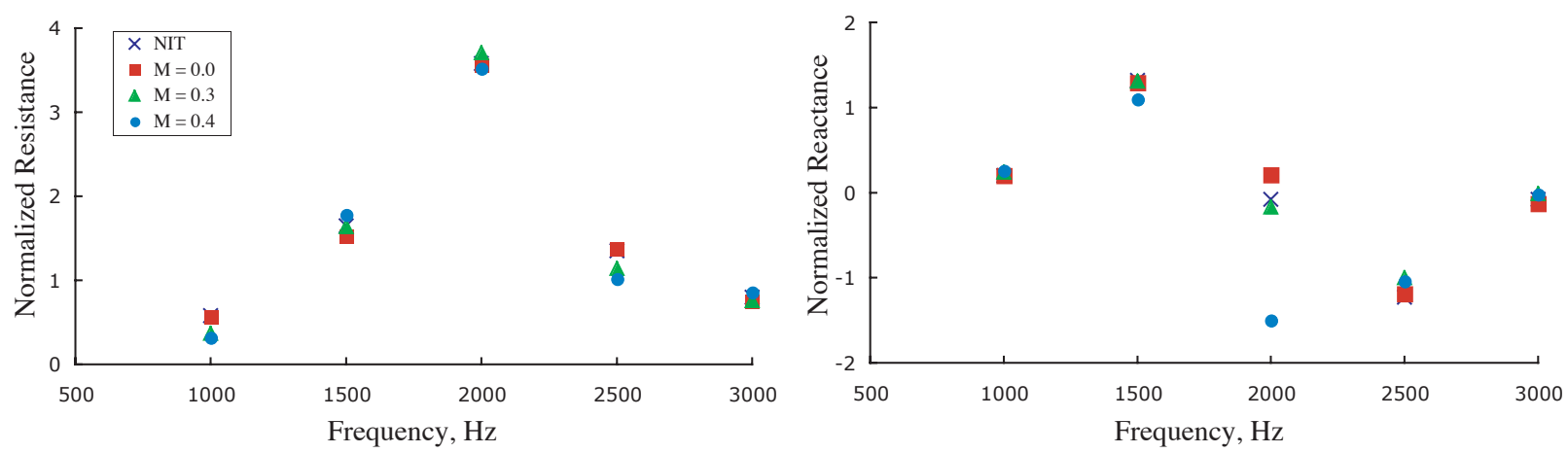

Figure 7. Ceramic tubular liner impedance $(\xi=\theta+i \chi)$. 

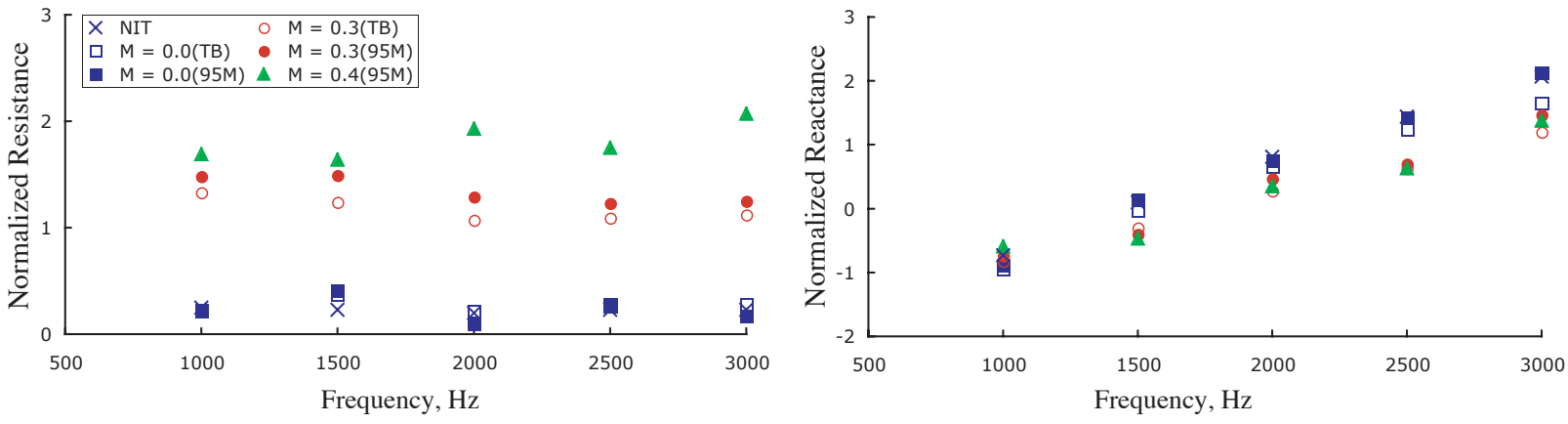

Figure 8. Conventional liner \# 1 impedance $(\zeta=\theta+i \chi)$.
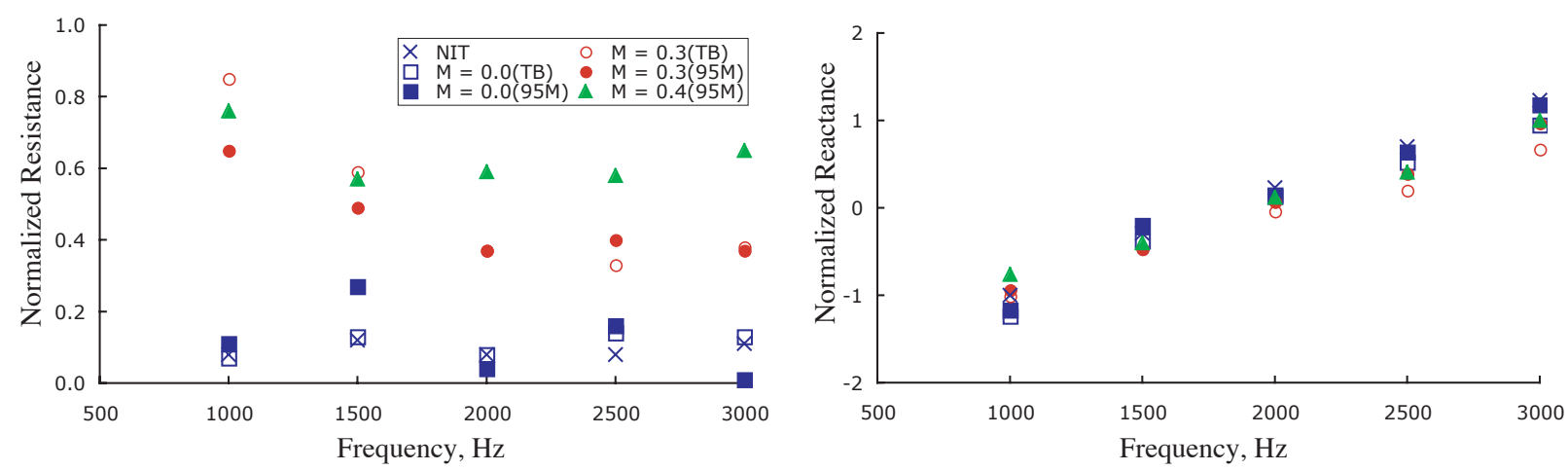

Figure 9. Conventional liner \# 2 impedance $(\zeta=\theta+i \chi)$.

\section{Results and Discussion}

$\mathrm{R}$ ESULTS for three acoustic liners are presented in the following section. Ceramic tubular liner impedances were educed in the GIT-95M to establish confidence in the data acquisition and post-processing methodology. Two conventional liners were also tested in the GIT-95M, and the impedances from these tests are compared to corresponding impedances educed using the GIT-TB.

The ceramic tubular liner test was used as a benchmark test for the system, to evaluate the new data acquisition and post-processing system (including analysis). Given the mean flow insensitivity of the ceramic tubular liner, impedances educed using data acquired at multiple mean flow velocities in the GIT-95M should be well matched to those educed in normal incidence tube (NIT) tests of the same ceramic tubular material. Results for this liner are presented in figure 7 and are listed in table 2. The three solid symbols (square for $M=0.0$, triangle for $M=0.3$, circle for $M=0.4$ ) depict the impedance (normalized resistance, $\theta$, in the upper chart, and normalized reactance, $\chi$, in the lower chart) spectra educed for the ceramic tubular liner using the 2-D FEM. Impedances educed with the same material in the Langley normal incidence tube are depicted with the ' $x$ ' symbols. As expected, there is excellent agreement between the impedances educed using the FEM (at all three mean flow velocities) and the corresponding values educed using NIT measurements. The only significant exception to this is at $2000 \mathrm{~Hz}$, which is near the anti-resonance frequency for this liner, where the normalized reactance varies from -1.5 to 0.2 . Due to the sensitivity of the impedance near antiresonance, this variability is to be expected. Otherwise, the excellent agreement, for $0.3 \leq \theta \leq 3.7$ and $-1.5 \leq \chi \leq 1.3$, provides confidence that the GIT-95M data acquisition and post-processing is functioning properly. 
Table 2. Impedances for ceramic tubular liner (educed using NIT and GIT-95M).

Normalized Resistance, $\theta$

\begin{tabular}{|c||c||c|c|c|}
\hline Freq, $\mathrm{Hz}$ & NIT & $M=0.0$ & $M=0.3$ & $M=0.4$ \\
\hline 1000 & 0.58 & 0.57 & 0.37 & 0.32 \\
\hline 1500 & 1.65 & 1.53 & 1.64 & 1.78 \\
\hline 2000 & 3.58 & 3.56 & 3.71 & 3.52 \\
\hline 2500 & 1.35 & 1.38 & 1.14 & 1.02 \\
\hline 3000 & 0.80 & 0.75 & 0.76 & 0.86 \\
\hline
\end{tabular}

Normalized Reactance, $\chi$

\begin{tabular}{|c||c||c|c|c|}
\hline Freq, $\mathrm{Hz}$ & NIT & $M=0.0$ & $M=0.3$ & $M=0.4$ \\
\hline 1000 & 0.20 & 0.20 & 0.24 & 0.26 \\
\hline 1500 & 1.32 & 1.29 & 1.31 & 1.10 \\
\hline 2000 & -0.08 & 0.21 & -0.17 & -1.50 \\
\hline 2500 & -1.23 & -1.19 & -1.00 & -1.04 \\
\hline 3000 & -0.08 & -0.13 & -0.01 & -0.02 \\
\hline
\end{tabular}

Table 3. Impedance results for conventional liner 1.

Normalized Resistance, $\theta$

\begin{tabular}{|c||c||c|c||c|c|c|}
\hline Freq, $\mathrm{Hz}$ & NIT & TB, $M=0.0$ & $\mathrm{~TB}, M=0.3$ & $95 \mathrm{M}, M=0.0$ & $95 \mathrm{M}, M=0.3$ & $95 \mathrm{M}, M=0.4$ \\
\hline 1000 & 0.25 & 0.23 & 1.33 & 0.22 & 1.48 & 1.69 \\
\hline 1500 & 0.23 & 0.37 & 1.24 & 0.41 & 1.49 & 1.64 \\
\hline 2000 & 0.20 & 0.22 & 1.07 & 0.10 & 1.29 & 1.93 \\
\hline 2500 & 0.23 & 0.26 & 1.09 & 0.28 & 1.23 & 1.75 \\
\hline 3000 & 0.23 & 0.28 & 1.22 & 0.17 & 1.25 & 2.07 \\
\hline
\end{tabular}

Normalized Reactance, $\chi$

\begin{tabular}{|c||c||c|c||c|c|c|}
\hline Freq, $\mathrm{Hz}$ & $\mathrm{NIT}$ & $\mathrm{TB}, M=0.0$ & $\mathrm{~TB}, M=0.3$ & $95 \mathrm{M}, M=0.0$ & $95 \mathrm{M}, M=0.3$ & $95 \mathrm{M}, M=0.4$ \\
\hline 1000 & -0.73 & -0.94 & -0.82 & -0.89 & -0.74 & -0.59 \\
\hline 1500 & 0.10 & -0.03 & -0.30 & 0.14 & -0.40 & -0.47 \\
\hline 2000 & 0.81 & 0.66 & 0.28 & 0.75 & 0.47 & 0.35 \\
\hline 2500 & 1.44 & 1.24 & 0.63 & 1.42 & 0.70 & 0.63 \\
\hline 3000 & 2.07 & 1.65 & 1.20 & 2.13 & 1.47 & 1.38 \\
\hline
\end{tabular}

Tables 3 and 4 contain impedances $(\xi=\theta+i \chi)$ educed with the 2-D FEM, for both conventional liners, using complex acoustic pressure data acquired in the GIT-TB and GIT-95M, respectively, for centerline Mach numbers of $0.0,0.3$ and 0.4 . These impedances are also presented in figures 8 and 9 . In these figures, the solid and open symbols depict the impedances educed in the new (GIT-95M) and old (GIT-TB) test sections, respectively. In addition, impedances educed using the Langley NIT are depicted with ' $\mathrm{x}$ ' symbols. As expected, the $M=0.0$ GIT impedances are observed to be well matched to the NIT impedances. However, the impedances educed with the GIT-TB and GIT-95M are clearly more dependent on the mean flow velocity.

In general, comparisons of impedances educed with the two test sections are very consistent. For the no-flow condition, there is good agreement between the two sets of results. With flow $(M=0.3)$, the trends are well matched, but the resistances are not quite as well matched as for the no-flow condition. The resistances for the first conventional liner are observed to be higher than the corresponding resistances for the second liner. Recall from table 1 that the 
Table 4. Impedance results for conventional liner 2.

Normalized Resistance, $\theta$

\begin{tabular}{|c||c||c|c||c|c|c|}
\hline Freq, $\mathrm{Hz}$ & $\mathrm{NIT}$ & $\mathrm{TB}, M=0.0$ & $\mathrm{~TB}, M=0.3$ & $95 \mathrm{M}, M=0.0$ & $95 \mathrm{M}, M=0.3$ & $95 \mathrm{M}, M=0.4$ \\
\hline 1000 & 0.08 & 0.07 & 0.85 & 0.11 & 0.65 & 0.76 \\
\hline 1500 & 0.12 & 0.13 & 0.59 & 0.27 & 0.49 & 0.57 \\
\hline 2000 & 0.08 & 0.08 & 0.37 & 0.04 & 0.37 & 0.59 \\
\hline 2500 & 0.08 & 0.14 & 0.33 & 0.16 & 0.40 & 0.58 \\
\hline 3000 & 0.11 & 0.13 & 0.38 & 0.01 & 0.37 & 0.65 \\
\hline
\end{tabular}

Normalized Reactance, $\chi$

\begin{tabular}{|c||c||c|c||c|c|c|}
\hline Freq, $\mathrm{Hz}$ & $\mathrm{NIT}$ & $\mathrm{TB}, M=0.0$ & $\mathrm{~TB}, M=0.3$ & $95 \mathrm{M}, M=0.0$ & $95 \mathrm{M}, M=0.3$ & $95 \mathrm{M}, M=0.4$ \\
\hline 1000 & -1.00 & -1.24 & -1.01 & -1.17 & -0.94 & -0.76 \\
\hline 1500 & -0.29 & -0.38 & -0.47 & -0.20 & -0.47 & -0.40 \\
\hline 2000 & 0.23 & 0.15 & -0.04 & 0.13 & 0.07 & 0.12 \\
\hline 2500 & 0.70 & 0.52 & 0.20 & 0.64 & 0.39 & 0.41 \\
\hline 3000 & 1.23 & 0.95 & 0.67 & 1.18 & 0.97 & 1.00 \\
\hline
\end{tabular}

porosity of conventional liner 1 is $6.4 \%$, as compared to a porosity of $15 \%$ for conventional liner 2 . Higher resistances for the lower porosity liner are to be expected. Given the number of differences between the measurement processes implemented with the two test sections (similar to conducting the tests in totally different facilities; see "GIT Test Sections"), together with minor flow field differences (differences in leakage and boundary layer growth), this level of agreement is believed to be acceptable. Finally, data acquired at $M=0.4$ in the GIT-95M are provided for comparison. As expected, the resistances at $M=0.4$ are generally higher than for $M=0.3$.

\section{Concluding Remarks}

$\mathrm{E}^{x}$ XTENSIVE modifications to the NASA Langley Grazing Incidence Tube have been completed, and initial results are presented for tests with three acoustic liners. Results for a ceramic tubular liner are used to demonstrate that the modified test section can be confidently used to acquire impedances (well matched with impedances educed in a normal incidence tube) over a significant range of impedances, for mean flow velocities up to at least $M=0.4$.

In addition, impedances educed for two conventional liners (perforate facesheet over honeycomb core) using the modified grazing incidence tube test window were compared with corresponding impedances educed using the original test window. These comparisons indicate that the modified test section can be confidently used to study liners using the 2-D finite element method previously developed for the original test section. This modified test section, together with an improved data acquisition system, is also demonstrated to provide an efficiency increase by at least a factor of five as compared with the previous test section.

Additional studies are planned to fully evaluate the new data acquisition and analysis system. Some of these are provided in the list below:

1. Further studies will be conducted with the current suction devices, as well as with blowing devices, such that the effects of boundary layer shape can be fully evaluated.

2. Multiple tests with the same liners will be conducted to study the repeatability of the system (data acquisition, post-processing and analysis).

3. The 3-D finite element method will be further developed, and will be used to study the effects of mean flow profiles, variable-impedance liners and higher-order modes. 


\section{References}

${ }^{1}$ Watson, W.R., Robinson, J.H., Jones, M.G., and Parrott, T.L., "Design and Attenuation Properties of Periodic Checkerboard Liners," AIAA2003-3309, "Proceedings of the 9th AIAA/CEAS Aeroacoustics Conference \& Exhibit," May 12-14, 2003.

${ }^{2}$ Alonso, J.S., Burdisso, R.A., "Sound Radiation from the Boundary in a Circular Lined Duct with Flow," AIAA-2003-3144, "Proceedings of the 9th AIAA/CEAS Aeroacoustics Conference \& Exhibit," May 12-14, 2003. 1061.

${ }^{3}$ Ingard, U., "On the Theory and Design of Acoustic Resonators," Journal of Acoustical Society of America, Vol. 25, No. 6, 1953, pp. 1037-

${ }^{4}$ Melling, T.H., "The Acoustic Impedance of Perforates at Medium and High Sound Pressure Levels," Journal of Sound and Vibration, Vol. 29, No. 1, 1973, pp. 1-65.

${ }^{5}$ Malmary, C., Carbonne, S., Auregan, Y., and Pagneux, V., "Acoustic Impedance Measurement with Grazing Flow," AIAA Paper 2001-2193, "Proceedings of the 7th AIAA/CEAS Aeroacoustics Conference \& Exhibit," May, 2001.

${ }^{6}$ Armstrong, D.L., Beckemeyer, R.J., and Olsen, R.F., "Impedance Measurements of Acoustic Duct Liners With Grazing Flow," Paper presented at the 87th Meeting of the Acoustical Society of America, New York, NY, 1974.

${ }^{7}$ Watson, W.R., Tanner, S.E., and Parrott, T.L., "Optimization Method for Educing Variable-Impedance Liner Properties," AIAA Journal, Vol. 36, No. 1, 1998, pp. 18-23.

${ }^{8}$ Jones, M.G., Parrott, T.L., and Watson, W.R., "Comparison of Acoustic Impedance Eduction Techniques for Locally-Reacting Liners," AIAA Paper 2003-3306, "Proceedings of the 9th AIAA/CEAS Aeroacoustics Conference \& Exhibit," May 12-14, 2003.

${ }^{9}$ Jones, M.G., Watson, W.R., Tracy, M.B., and Parrott, T.L., "Comparison of Two Waveguide Methods for Educing Liner Impedance in Grazing Flow," AIAA Journal, Vol. 42, No. 2, 2004, pp.232-240.

${ }^{10}$ Bendat, J.S. and Piersol, A.G., "Random Data: Analysis and Measurement Procedures," Wiley-Interscience, 1971.

${ }^{11}$ Jones, M.G. and Stiede, P.E., "Comparison of Methods for Determining Specific Acoustic Impedance," Journal of Acoustical Society of America, Vol. 101, No. 5, 1997, pp. 2694-2704.

${ }^{12}$ Watson, W.R., Tracy, M.B., Jones, M.G. and Parrott, T.L., "Impedance Eduction in the Presence of Shear Flow," AIAA 2001-2236, "Proceedings of the 7th AIAA/CEAS Aeroacoustics Conference \& Exhibit," May, 2001.

${ }^{13}$ Myers, M.K., "On the Acoustic Boundary Condition in the Presence of Flow," Journal Of Sound And Vibration, Vol. 71, No. 3, 1980, pp. 429-434.

${ }^{14}$ Stewart, G.W. III, “A Modification of Davidon's Minimization Method to Accept Difference Approximations of Derivatives,” Journal of ACM, Vol 14, No. 1, 1967, pp 72-83. 